\title{
Diseño y sociedad: teorías y definiciones
}

Veronica De Salvo ${ }^{1}$

Curso de Laurea en Diseño Industrial Universidad de Florencia

\section{Resumen}

El conjunto de herramientas, métodos y áreas temáticas, que a lo largo del tiempo han caracterizado la disciplina del diseño, demuestran la existencia de un vínculo estrecho entre el diseño y las transformaciones sociales, económicas, políticas y culturales ocurridas en diferentes momentos históricos. A través de un análisis temático de estos eventos en este artículo, se describen las diversas etapas de la evolución del diseño, destacando cómo las diferentes dinámicas histórico-sociales han influido en la cultura del diseño y el papel del diseñador, desde su aplicación a la producción industrial hasta la construcción y activación de procesos de innovación social.

Palabras clave: evolución del diseño; transformaciones sociales; diseño industrial; diseño social; diseño ambiental; diseño para la innovación social; educación en diseño; escenarios futuros.

\section{Design and society: theories and definitions}

\begin{abstract}
The set of tools, methods and thematic areas that over time have characterized the discipline of design, demonstrates the existence of a close link between design and the social, economic, political and cultural transformations occurred in different historical moments. Through a thematic analysis of these events in this article the various stages of the evolution of design are described, highlighting how the different historical-social dynamics have influenced the design culture and the role of the designer, starting from the design for industrial production to the construction and activation of social innovation processes.
\end{abstract}

Keywords: design evolution; social transformations; industrial design; social design; environmental design; design for social innovation; education in design; future scenarios.

\footnotetext{
1 Doctora en Medio Ambiente, Diseño e Innovación por la Universidad de la Campania "Luigi Vanvitelli", Departamento de Ingeniería Civil, Diseño, Construcción y Medio Ambiente (DICDEA). Master en Diseño Social por la Design Academy Eindhoven. Licenciada en Diseño Industrial por la Universidad de Palermo. Ganadora de una beca de investigación para "Diseño entre fabricación 4.0 e Innovación social" por la Universidad de Florencia, Departamento de Arquitectura (DIDA).

E-mail: veronica.desalv089@.gmail.com
} 
El camino histórico de la disciplina del diseño se ha caracterizado por la alternancia de modelos teóricoprácticos diferentes, y en muchos casos opuestos, que han marcado significativamente la evolución de este ámbito de la cultura del proyecto, contribuyendo a una redefinición continua del papel que, a través de su propia profesió, el diseñador ha interpretado dentro de los diferentes contextos histórico-sociales y la caracterización social y cultural de la disciplina.

En un intento perpetuo de relacionar los aspectos técnico-productivos con las dinámicas socioculturales de la contemporaneidad de referencia, el diseño siempre ha identificado nuevos temas y nuevos caminos de entrenamiento en relación con su misión de diseño, pasando de su estrecha conexión inicial con el mundo de la industria y los procesos de producción a nuevas expresiones del producto determinadas por los cambios en la cultura y las necesidades del consumidor, hasta representar un factor crucial en la activación de los procesos más recientes de innovación social y ambiental.

Es indudable, por lo tanto, que la relación espacio-tiempo en la que ha evolucionado el diseño, durante las fases de su desarrollo, ha influido cada vez en los contenidos y en las metodologías de diseño, fortaleciendo la conciencia de las responsabilidades que la actividad del proyecto asume en relación con la realidad contemporánea (Trapani, 2008, p. 15).

\section{El Diseño entre arte, industria y artesanía}

Recordando las primeras teorías del diseño, es posible rastrear el debate ideológico sobre la relación entre el arte y la industria, que interesó a la cultura europea de finales del siglo XIX, en la cual la posición anti industrial y antiurbana expresada por John Ruskin y William Morris ${ }^{2}$ se opuso a la idea explícitamente favorable a la industrialización declarada por los fundadores del Deutscher Werkbund (1907), una asociación establecida para salvaguardar y mejorar la calidad de los objetos de uso de fabricación alemana a través de la colaboración entre arte, industria y artesanía.

Esta primera fase será secundada, a partir de 1919, por Walter Gropius y la práctica de la Bauhaus, que a través de un vínvulo experimental entre investigación artística e investigación tecnológica promovió un nuevo método didáctico y de proyecto centrado en el diseño del producto industrial. Esta metodología, caracterizada por un código formal específico, a pesar de su singularidad terminó asumiendo un carácter tan rígido -como lo denunció László Moholy-Nagy uno de los principales teóricos de la escuela- que disminuyó la fase exploratoria del proceso a favor del resultado final (Branzi, 2008).

En 1946, en continuidad con las estrategias de la Bauhaus, nació la Ulm Hochschule für Gestaltung, cuya 
gestión se encomendó primero a Max Bill y luego, en 1956, a Tomás Maldonado. Este último considera el diseño como una profesión fundada científicamente y dirige la escuela hacia la gran producción industrial en serie, a través de una estrategia educativa basada en metodologías de diseño racionalmente definibles y repetibles, dirigida a la realización de productos técnicos funcionalmente perfectos y ergonómicos. De esta manera, el diseño se convirtió en un elemento constitutivo de la lógica racional de la producción, en total armonía con la cultura mecánica y el mercado de consumo que caracterizó este período histórico.

\section{El Diseño como fenómeno social total}

Al analizar la evolución del diseño, a través de un análisis centrado principalmente en los temas abordados por el proyecto, en relación con el contexto cambiante de referencia y el pensamiento que formó la base de esto, se decidió dar un salto en el tiempo para reconectarse con el ya citado Maldonado. En su ensayo de 1976 describe el diseño como fenómeno social total, que debe considerarse siempre en relación con otros fenómenos con los que constituye un solo tejido conectivo, con una hipotesis de futuro en que el diseño industrial es un catalizador para una mayor participación entre diferentes fuerzas productivas.

Esta dimensión social del diseño y la responsabilidad ética que se deriva de ella habían sido ampliamente abordadas hace algunos años por Viktor Papanek. Éste apoya la fuerte conexión entre el diseño y el "mundo real", en su libro titulado Diseñar para el mundo real invitó a la figura del diseñador a investigar nuevas áreas, más allá de las clásicas de diseño formal y funcional, para responder a los desafíos impuestos por la sociedad contemporánea. Convencido de que la actividad de proyecto de los diseñadores puede producir cambios profundos en el mundo, Papanek subraya la importancia de una "vocación moral" por parte del diseñador hacia la sociedad y, aún más importante, de la conciencia de los impactos que su acción puede tener en el cambio social (Papanek, 1971) ${ }^{3}$.

En los mismos años setenta, Gui Bonsiepe explicó la relación entre el diseño y la política a través de la relevancia social que se reconoce al proyecto, definiendo como crucial el papel de la disciplina del diseño para la producción de la realidad ${ }^{4}$ y afirmando que «(..) la utopía social sin instrumentación técnica se reduce a la mera declamación: permanece en el verbalismo ambicioso en lugar de traducirse en acción realista. No hay heroísmo en la derrota». (Bonsiepe, 1975, p. 10). Además formuló la hipótesis de sobre en qué medida la actividad del proyecto podría actuar como una clave para el desarrollo en los países no industrializados, renunciando a su función de embellecer el status quo determinado por el aumento en la circulación de mercancías en respuesta a las necesidades de la metrópolis.

Una síntesis de estas teorías se puede rastrear en la actividad de Riccardo Dalisi, quien entre 1971 y 1975 experimentó con una serie de experiencias en las que se incluyeron niños y jóvenes del barrio de Traiano en Nápoles (fig.1), caracterizado por una alta tasa de abandono escolar, a través de acciones de planificación participativa (otras seguirán con los niños de Secondigliano, Ponticelli, Sanità, Scampia y Nisida). Con Dalisi, el diseñador ya no está únicamente influenciado por la sociedad, sino que encaja totalmente dentro de su tejido urbano y cultural, interceptando los aspectos más problemáticos.

3 «Design must become an innovative, highly creative, cross-disciplinary tool responsive to the true needs of men. It must be more research oriented and we must stop defiling the earth itself with poorly-designed objects and structures».

4 «L'operare tecnologico, che comprende anche il disegno industriale, partecipa in modo mediato al processo di trasformazione sociale, tramite la prassi di ogni disciplina specifica». 


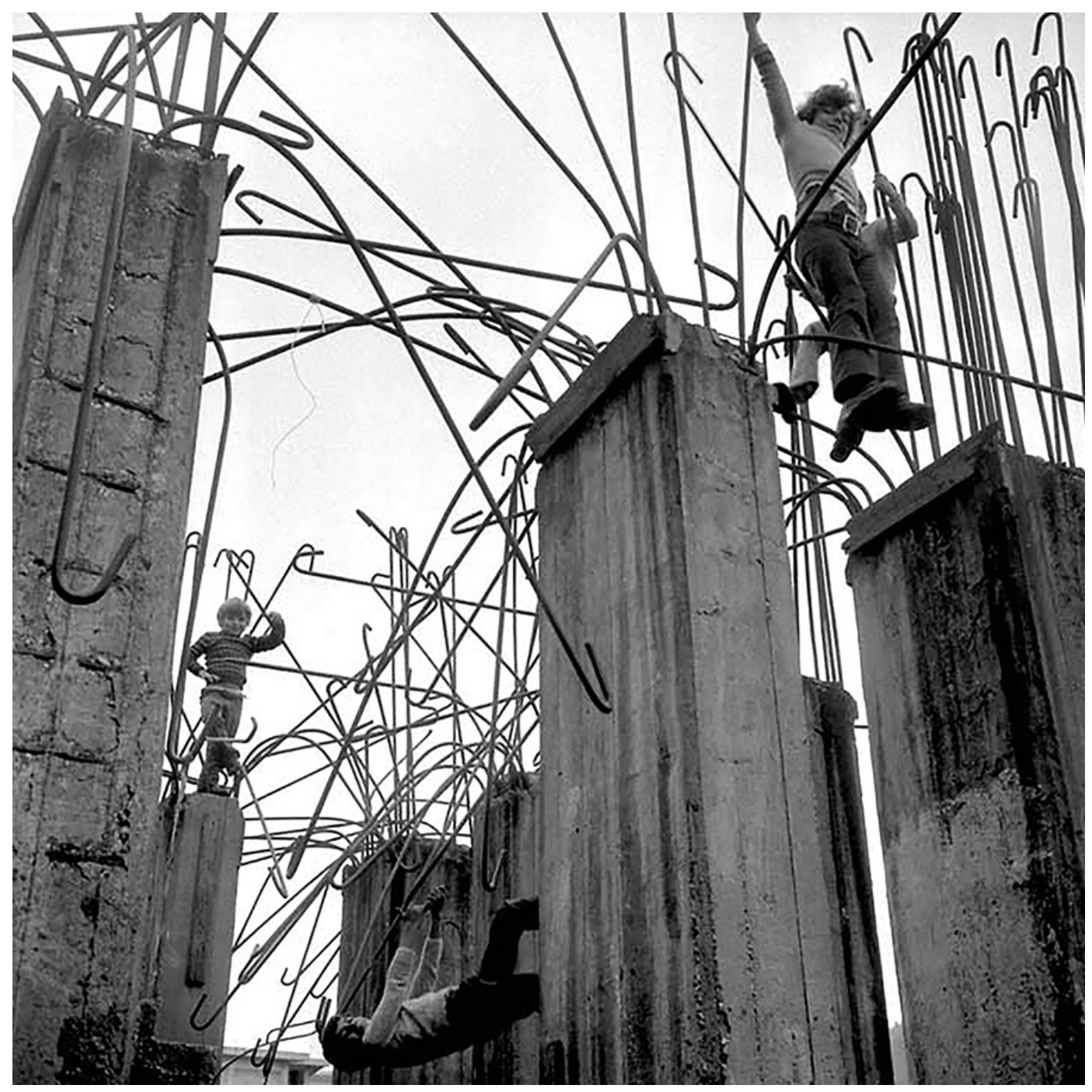

Fig. 1. Animación en el distrito de Traiano, c. 1974.

Entre los autores que reconocieron el papel activo del diseñador dentro de la sociedad también se encuentra Andrea Branzi, quien va más allá al afirmar el derecho del diseñador a promover un punto de vista crítico hacia los desafíos a que se enfrenta en el transcurso de su actividad. De hecho, afirma que en un contexto en el que la globalización ha producido profundas transformaciones sociales y urbanas, el diseño está llamado a modificar sus bases operativas, dejando de ser «una actividad pura y simple destinada a responder a un mercado exclusivo de muebles sofisticados, para convertirse en una de las actividades fundamentales para el funcionamiento de la ciudad contemporánea, que prevé una constante inestabilidad de sus funciones» (Branzi, 2008). Branzi respalda la capacidad del diseño para inventar relaciones e intercambios (incluso virtuales) dentro de una sociedad generalizada, sin comprometerse a «buscar soluciones y productos definitivos destinados a largo tiempo, pero en cambio creando procesos ininterrumpidos de innovación tecnológica, mercadería y estética, funcional o comercial, para un mercado inquieto y turbulento, que sigue lógicas selectivas ya menudo impredecibles» (Branzi, 2008). 


\section{El Diseño para la sostenibilidad ambiental}

El desarrollo de nuevos modelos de consumo también ha dado lugar a la aparición de una nueva especificación del diseño, definible con los términos de ecodiseño o diseño para la sostenibilidad ambiental. De hecho, a lo largo de las décadas ha habido una intensa reflexión sobre los problemas ambientales y la posibilidad de desarrollar modelos y prácticas sostenibles. En este contexto, el trabajo de los diseñadores se centró en la definición de estrategias de diseño capaces de permitir la reducción de los recursos utilizados en los procesos de producción, además de la prolongación de la vida de los objetos y la renovación de los materiales.

En relación con este tema, es posible repasar la evolución histórica de la cultura del diseño que, ya en el siglo XIX, encontró en Morris uno de los principales críticos de la degradación ambiental generada por la expansión de la producción industrial. Posteriormente durante el siglo XX varios diseñadores, entre quienes estaba Alvar Aalto, concentraron sus actividades de selección y procesamiento de materiales con respecto al medio ambiente o, en un intento por contaminar menos, se comprometieron en proyectos sostenibles y democráticos, como lo demuestran también las experiencias de autoproducción de Enzo Mari. Además, en los años setenta, el mismo Maldonado denunció el maltrato progresivo del entorno físico, apoyando la necesidad de recuperar una actitud racional que él define como "esperanza proyectual".

En los años noventa, a la luz de la Conferencia de Río (1992) y el Protocolo de Kyoto (1997), en relación a temas de materia ambiental, vemos el desarrollo de una estrategia de proyecto destinada a definir los factores de control y la eficiencia de las tecnologías, de los materiales y de los productos. Todo ello en relación con las oportunidades de mejoría que este enfoque puede aportar a nuestra calidad de vida. Con respecto a los aspectos ambientales, de los cuales la actividad proyectual debe tener en cuenta, Medardo Chiapponi también respondió proponiendo la idea de una visión sistémica del problema ambiental e identificando algunas soluciones posibles (Chiapponi, 1989, p. 27) ${ }^{5}$.

La definición de escenarios futuros sostenibles fue puesta en escena en la exposición /l giardino delle cose, curada por Ezio Manzini y presentada en la Trienal de Milán en 1992, en la que se propone la reversión de las restricciones ambientales, para que se traduzcan en nuevas oportunidades para la actividad del diseño que, sobre la base de nuevos parámetros de calidad, ahora puede avanzar en la propuesta de soluciones diferentes e innovadoras.

\section{El Diseño para la Innovación Social}

Es el mismo Manzini quien en uno de sus trabajos más recientes respalda, reconectándose con lo que ha sido afirmado por Chiapponi y algunos de los autores mencionados anteriormente, la necesidad de que el diseño trabaje a nivel sistémico liberándose de un diseño puramente estético y funcional de productos para consumo y dirigiéndose hacia nuevos procesos de innovación que, además de la sostenibilidad ambiental, también incluyan la sostenibilidad que podríamos definir de carácter social.

5 «Siamo ben lontani -egli sostiene- da una natura primordiale, immutevole ed estranea. Ci troviamo piuttosto di fronte a un sistema ambientale destinato a trasformarsi e a mutare continuamente per effetto delle azioni e delle reazioni che si svolgono tra le parti che lo costituiscono e tra ognuna di esse e la totalità». 
El Diseño para la Innovación Social está, de hecho, descrito por el autor como una herramienta útil para mejorar la vida de las personas mediante la generación de procesos de cambio a través de la recombinación creativa de elementos existentes, previamente desconectados o aplicados en diferentes áreas (Manzini, 2015). Por lo tanto, dentro del contexto social, el diseñador puede operar de manera innovadora planificando con las comunidades, y promoviendo así la participación y colaboración entre los diferentes actores involucrados en la definición de los escenarios del proyecto, o para las comunidades, comprometiéndose a desarrollar productos y servicios capaces de mejorar la accesibilidad, la eficiencia y la reproducibilidad de los contextos específicos. Sobre la base de estas consideraciones, Manzini afirma que la posibilidad real del cambio social consiste en generar una conversación social entendida como una actividad de co-design en la que los participantes y los expertos pueden colaborar, contribuyendo conjuntamente al proceso de cambio (Manzini, ibidem).

\section{El papel de las escuelas de Diseño}

Como sucedió en el pasado con la Bauhaus y la Escuela de Ulm, hoy en el debate que ha visto evolucionar las diferentes posiciones, siempre actualizadas de la cultura del proyecto, se insertan las rutas de aprendizaje y los experimentos desarrollados en las escuelas contemporáneas a través de nuevos métodos pedagógicos que han contribuido a la continua redefinición del concepto de diseño.

En relación con lo que afirmó Stefano Maffei, según el cual «El diseño ya no puede posponer una consideración sobre recursos como la energía, la biodiversidad, los desafíos ambientales y humanos, los servicios, la comunicación y la interacción» (Maffei, 2014, p. 130), el Politécnico de Turín, por ejemplo, ha desarrollado a lo largo de los años una metodología basada en un enfoque sistémico para el diseño y la producción, que permite gestionar cuantitativamente y cualitativamente cada etapa del proceso de producción, coordinando y verificando las relaciones con otros ciclos de producción y evitando así todo tipo de residuos.

Mirando hacia atrás, a todo el panorama europeo, hay diferentes escuelas en Europa -Royal College of Art, Central Saint Martins, Ecal, por nombrar algunas- que a través de la aplicación de metodologías transdisciplinarias han trabajado en los temas e historias de la realidad contemporánea. Ello ha dado lugar a resultados capaces de traducir los desafíos actuales en escenarios futuros mejores y plausibles. Entre estas, la Design Academy Eindhoven representó un caso único en Europa, hasta hace unos años, gracias a la oferta de un curso de estudio centrado en el enfoque del Diseño Social (fig.2) en el que la actividad del proyecto conectada con los temas considerados en relación con los contextos sociales de referencia y la originalidad, a menudo provocativa, expresada por los trabajos de los estudiantes, han constituido un rasgo distintivo de los resultados producidos. Esto ha permitido la afirmación de una nueva figura del diseñador capaz de encajar, como otros profesionales, en áreas temáticas para las cuales la acción del diseño aún no había sido hipotetizada.

Estas experiencias también confirman que delante de las transformaciones que tienen lugar en varios frentes y sin precedentes por velocidad, escala e intensidad, el diseño se puede utilizar como una herramienta de conciencia 'política', o como un medio para discutir posibles o mejores futuros. 


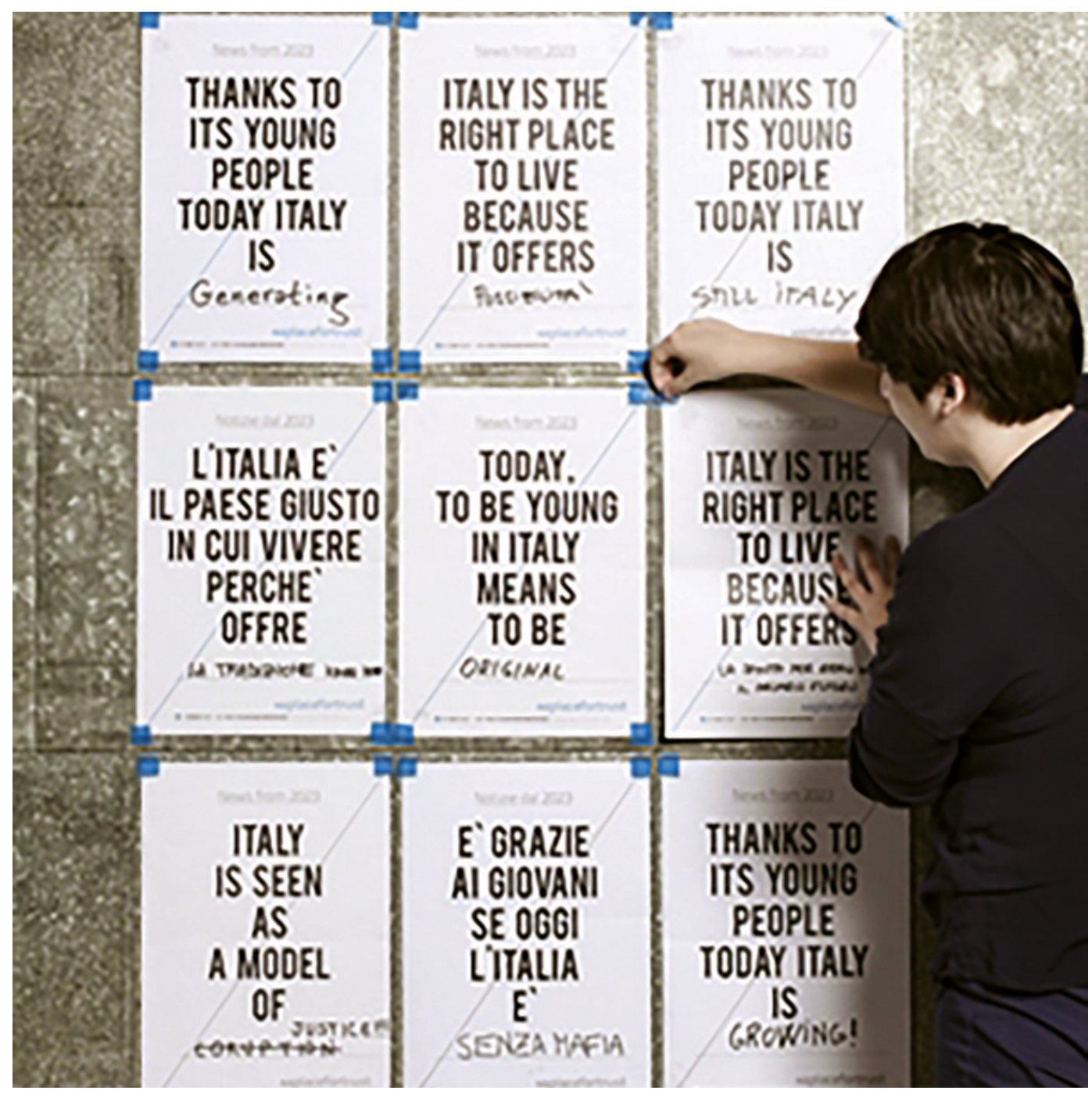

Fig. 2. A place for Trust, Social Design Master por la Design Academy Eindhoven, 2014.

\section{Escenarios futuros para el Diseño}

En conclusión, en un intento de predecir lo que se puede esperar del diseño del mañana, es estimulante reanudar lo que afirmó John Thackara, quien en 1998 hipotetizó un escenario futuro que en 2028 el proyecto será inseparable del contexto y el diseñador jugará un papel de liderazgo en los procesos de cambio de la sociedad, actuando dentro de un proceso colectivo de innovación y realizando no solo productos, sino ideas, conocimientos, procesos y relaciones «a través de la experiencia de proyectos reales, dentro de comunidades reales, con empresas reales y utilizando nuevas tecnologías» (Thackara, 1998).

El diseñador ya no puede limitarse a acciones con un carácter neutral, sino que está llamado a hacer uso de sus herramientas disciplinarias para definir nuevas formas de desarrollo de los procesos de transformación y organización de la sociedad, a través de la realización de proyectos dirigidos explícitamente a activar nuevas relaciones socio-espaciales. 
Sobre la base de estas consideraciones, se requiere el empeño del diseñador para hacer frente a las nuevas cuestiones éticas que afectan a la realidad política, social y económica actual. Sin ello se crearía una situación de total ausencia de responsabilidad, como ha declarado Vilém Flusser al afirmar que «Si tenemos éxito, más allá de todas las ideologías, para encontrar una manera de abordar una solución de los problemas éticos en la fase de diseño, entonces el nazismo, la guerra en el Golfo y eventos similares pasarán a la historia como las etapas iniciales de un período de destrucción y autodestrucción. El hecho mismo de que comencemos a hacernos estas preguntas es una fuente de esperanza» (Flusser, 2003, p. 67).

\section{Bibliografía}

Alessi C. (2014). Dopo gli anni Zero. Il nuovo design italiano (intervista a Stefano Maffei). Bari: Laterza.

Bonsiepe G. (1975). Teoria e pratica del disegno industriale. Elementi per una manualistica critica. Milano: Feltrinelli.

Branzi A. (2008). Design. Il libro dell'anno 2008. Treccani.

Chiapponi M. (1989). Ambiente: gestione e strategia. Un contributo alla teoria della progettazione ambientale. Milano: Feltrinelli.

Flusser V. (2003). Filosofia del design. Milano: Mondadori.

Fuad Luke A. (2009). Design Activism: Beautiful Strangeness for a Sustainable World. London: Rout-ledge.

Germak C. (curador). (2008). Uomo al centro del progetto. Design per un nuovo umanesimo. Torino: Allemandi. Maldonado T. (1976). Disegno industriale: un riesame. Milano: Feltrinelli.

Manzini E. (2015). Design. When Everybody Designs. MIT Press.

Papanek V. (1971). Design for the Real World: Human Ecology and Social Change. New York: Pantheon Books. Thackara J. (1998). 2028: fuga dall'entropia. Domus, núm. 800, enero.

Trapani V. (2008). Welcome to Design: riflessioni e percorsi di avvicinamento al progetto. Vicenza: Terra Ferma. 